\title{
Evidence for coherent collective Rydberg excitation in the strong blockade regime
}

\author{
Rolf Heidemann, ${ }^{1, *}$ Ulrich Raitzsch, ${ }^{1}$ Vera Bendkowsky, ${ }^{1}$ Björn \\ Butscher, ${ }^{1}$ Robert Löw, ${ }^{1}$ Luis Santos, ${ }^{2}$ and Tilman Pfau ${ }^{1, \dagger}$ \\ ${ }^{1}$ 5. Physikalisches Institut, Universität Stuttgart, Pfaffenwaldring 57, 70569 Stuttgart, Germany \\ ${ }^{2}$ Institut für Theoretische Physik, Universität Hannover, Appelstraße 2, 30167 Hannover, Germany
}

(Dated: June 19, 2007)

\begin{abstract}
Blockade effects on the single quantum level are at the heart of quantum devices like single-electron transistors. The blockade mechanisms are based on strong interactions like the Coulomb interaction in case of single electrons. Neutral atoms excited into a Rydberg state experience abnormally strong interactions that lead to the corresponding blockade effect for Rydberg atoms. In this paper we report on our measurements of a strong van der Waals blockade, showing that only one out of several thousand atoms within a blockade volume can be excited. In addition, our experimental results demonstrate the coherent nature of the excitation of magnetically trapped ultracold atoms into a Rydberg state, confirming the predicted dependence of the collective Rabi frequency on the square root of the mesoscopic system size. This collective coherent behaviour is generic for all mesoscopic systems which are able to carry only one single excitation quantum.
\end{abstract}

Early studies on atomic beams, where line broadening effects at high Rydberg densities were observed [1], triggered experiments on ultracold samples $[2,3,4,4,5,6,6,7,8$, 9, 10, 11], where the atomic motion of the atoms during the lifetime of the Rydberg atoms can be neglected. This excited state of matter is known as frozen Rydberg gas. The coherent elastic interaction between Rydberg atoms leads to a blockade effect which has been proposed as a crucial ingredient for rapid quantum gates either using single neutral atoms [12] or mesoscopic samples [13] to store and process quantum information. This blockade effect has been studied in various experiments using laser cooled atoms prepared in magneto-optical traps [2, 3, 4]. Typically the interaction effect was studied by changing the density of Rydberg atoms or by changing the principal quantum number $n$ of the excited Rydberg state. As the van der Waals interaction scales with $n^{11}$ a reduction of the excitation rates was observed for increasing $n$. A related interaction, the resonant dipole-dipole interaction, has been investigated in the last years [5, [6], usually tuned with an electric field but also with a magnetic field 7]. Many-body effects between some few atoms due to this interaction have been spectroscopically resolved 8] and their dependence on dimensionality was studied [9]. Recently, the first coherent excitations of non-interacting ultracold atoms into a Rydberg state have been achieved with the use of STIRAP sequences [10, 11]. Incoherent interactions could result in a decay to different Rydberg states, ionization or state changes by black body radiation.

In this paper we report on coherent Rydberg excitation of magnetically trapped ultracold atoms in the strong blockade regime. In this regime the excitation is strongly suppressed compared to the non-interacting case and limited to a maximum value which is in our experiment one out of few thousand ground state atoms. We confirm the collective nature of the coherent excitation by the dependence of the collective Rabi frequency on the square root of the mesoscopic system size. This size dependence is generic for all mesoscopic quantum systems for which the excitation is restricted to a single quantum, including socalled 'superatoms' recently discussed in the context of single photon storage [14].

A single atom exposed to resonant excitation light coherently oscillates with the single-atom Rabi frequency $\Omega_{0}$ between the ground and excited state. An ensemble of $N$ non-interacting atoms gives just $N$ times the singleatom Rabi-oscillation at frequency $\Omega_{0}$. But if for all members of the ensemble the interaction between atoms in the excited state is much stronger than the linewidth of the excitation, the ensemble can carry only one excitation. As the excitation can be located at any of the $N$ atoms this collective state is of the form:

$$
\left|\psi_{e}\right\rangle=\frac{1}{\sqrt{N}} \sum_{i=1}^{N}\left|g_{1}, g_{2}, g_{3}, \ldots, e_{i}, \ldots, g_{N}\right\rangle,
$$

where $g_{k}$ indicates an atom numbered $k$ in the ground state and $e_{i}$ one atom $i$ in the excited state. Therefore, the ensemble is excited collectively and oscillates with the collective Rabi frequency $\sqrt{N} \Omega_{0}$ between the ground state and the collectively excited state $\left|\psi_{e}\right\rangle[13$. In this sense the ensemble of $N$ atoms acts like a 'superatom' 14] with a transition dipole moment which is enhanced by a factor $\sqrt{N}$ as compared to the individual atoms.

The so-called blockade radius is defined as the interatomic distance where the interaction energy becomes equal to the linewidth of the excitation, which is in our experiments dominated by power-broadening (Fig.1b). We define the strong blockade regime by a blockade radius significantly larger than the mean interatomic distance, i.e. $N \gg 1$. In our experiments the sample size is larger than the blockade radius, we therefore model the sample by an ensemble of 'superatoms'. Additionally in our system, the density of ground state atoms and by this $N$, the atom number per 'superatom', is inhomogeneous. 
a

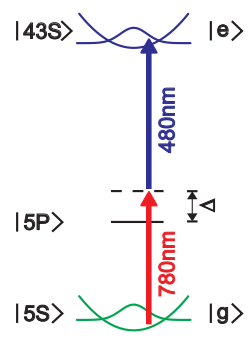

b

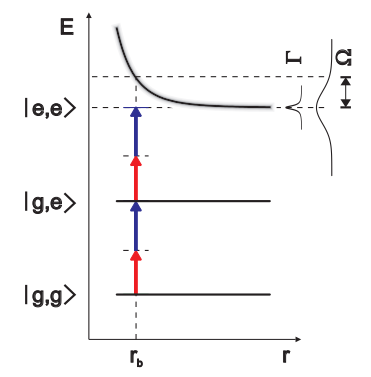

FIG. 1: a Two-photon excitation of magnetically trapped atoms from the $5 S_{1 / 2}, F=2, m_{F}=2$ state into the $43 S_{1 / 2}, m_{S}=1 / 2$. Due to the large detuning $\Delta$, the three levels are reduced to an effective two-level system $(|g\rangle,|e\rangle)$. $\mathbf{b}$ Molecular potential curves as a function of the relative coordinate $r$ (not to scale). The strong van der Waals interaction between the excited Rydberg states leads to a blockade effect for $|e, e\rangle$. Double excitation for distances smaller than the blockade radius $r_{\mathrm{b}}$ is strongly suppressed. For two atoms the blockade radius is either determined by the linewidth $\Gamma$ or the power broadening $\Omega$ (see Eq. 3).

With it the collective Rabi frequency is inhomogeneously distributed and the local oscillations add up to a total population that for short times increases quadratically. But after a very short time the Rydberg population shows a linear increase that falls behind the quadratic increase without interaction (see Fig.2k). This time is related to the inverse maximum collective Rabi frequency in the sample. For all experimental conditions shown in this paper this time is shorter than $50 \mathrm{~ns}$. For longer excitation times, the excitation in the strong blockade regime can be distinguished from single-atom behaviour by a strong suppression of excitation (see inset Fig.2a). The time scale for the subsequent linear increase is proportional to the inverse of the averaged collective Rabi frequency $\sqrt{N_{\text {mean }}} \Omega_{0}$. Due to the inhomogeneity the excited state population reaches after a time, that is also related to $\left(\sqrt{N_{\text {mean }}} \Omega_{0}\right)^{-1}$, a constant saturation value that is determined by the number of 'superatoms' in the sample. This number shows, as it will be explained below, a scaling with $\Omega_{0}$ and the density of ground state atoms, which is characteristic for the underlying blockade mechanism.

In our experiments we use magnetically trapped $\mathrm{Ru}-$ bidium atoms, evaporatively cooled to a few $\mu \mathrm{K}$. We investigated the excitation dynamics and observe full saturation for a large range of densities and excitation rates. The observed scaling of the initial increase and the saturation population with density and Rabi frequency provides evidence of coherent collective excitation as predicted by the 'superatom' model.

We start with a sample of $N_{\mathrm{g}}=1.5 \times 10^{7}{ }^{87} \mathrm{Rb}$ atoms in the $5 S_{1 / 2}, F=2, m_{F}=2$ state at a temperature of $3.4 \mu \mathrm{K}$ and a Gaussian density distribution with a peak value $n_{\mathrm{g}, 0}$ of $8.2 \times 10^{13} \mathrm{~cm}^{-3}$ in a specialized setup for

Rydberg experiments [15]. The excitation to the $43 S_{1 / 2}$ Rydberg state is done in a Ioffe-Pritchard-type trap with a two-photon transition via the $5 P_{3 / 2}$ state with a detuning $\Delta$ to the blue by $478 \mathrm{MHz}$ (see Fig.11a). We choose an $S$ state as it has only one repulsive branch in its molecular potential (see Fig.10), whereas higher $l$ states typically have repulsive and attractive branches and are subject to enhanced ion formation. Resonant dipole-dipole interaction due to the dominating transition $43 S+43 S \rightarrow$ $42 P+43 P$ is negligible for this experiment [16]. For the $5 S-5 P$ transition the Rabi frequency $\Omega_{1}$ was determined by Autler-Townes splitting at higher intensities [17]. In the current experiment, $\Omega_{1}$ is varied from $2.0 \mathrm{MHz}$ to 9.7 MHz. For the upper transition we estimate a Rabi frequency $\Omega_{2}$ of $21 \mathrm{MHz}$ from our calculation of the dipole matrix element. This gives a two-photon Rabi frequency $\Omega_{0}=\Omega_{1} \Omega_{2} /(2 \Delta)$ of up to $210 \mathrm{kHz}$. Due to the large detuning from the lower transition, the change in the density and momentum distribution due to absorption of photons is negligible [15]. The alignment of the excitation laser beams to the offset field of the magnetic trap together with the adjustment of polarizations makes it possible to preserve the magnetic moment and avoid energy shifts due to magnetic fields [15]. The waists of the Gaussian laser beams are large compared to the $1 / e^{2}$ radius of the sample and the Rabi frequency is almost constant over the sample [15].

During the experiment the excitation lasers are switched on for an excitation time $\tau$, which is varied between $100 \mathrm{~ns}$ and $20 \mu \mathrm{s}$. The longest excitation time is shorter than the $100 \mu$ s lifetime of the $43 S$ state [15, 18. Although the thermal motion of the ground state atoms is frozen out on the time scale of the excitation, attractive interaction between the Rydberg atoms can lead to collisions and ionization within this time scale [16, 19]. To avoid all effects of ions and electrons on the Rydberg atoms we chose a Rydberg state with repulsive van der Waals interaction $\left(C_{6}=-1.7 \times 10^{19}\right.$ a.u. [20] $)$ and applied an electric field of $200 \mathrm{~V} / \mathrm{m}$ strength during the excitation. With this field enhanced ionization by means of trapped electrons is suppressed [21], which would otherwise limit the lifetime 22]. Possibly produced ions are extracted from the sample within a time of $400 \mathrm{~ns}$, which is shorter than the time scales of the interactions of interest here. We resolved a density dependent blue shift of the excitation spectral line, which was not observed without field. This blue shift is a clear evidence for repulsive interaction whereas the electric field of charged particles would shift the spectroscopic lines to the red since the Stark shift is negative for this state. In the described experiments the excitation lasers are tuned to resonance, which was determined from an excitation spectrum. This was done with very low laser power and thus low Rydberg densities at which no line shift and broadening caused by interactions are observed. The linewidth of excitation was measured to be smaller than $130 \mathrm{kHz}$ on the 


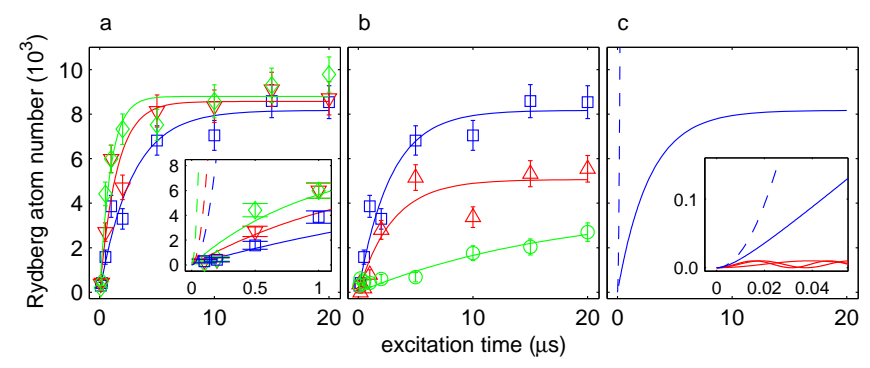

FIG. 2: a The Rydberg atom number plotted versus excitation time for a high laser intensity $\left(\Omega_{0}=210 \mathrm{kHz}\right)$ and three values of the density of ground state atoms $n_{\mathrm{g}, 0}=$ $\left(3.2 \times 10^{13}(\diamond), 6.6 \times 10^{12}(\nabla), 2.8 \times 10^{12}(\square)\right) \mathrm{cm}^{-3}$. b The Rydberg atom number plotted versus excitation time for a low atom density $\left(n_{\mathrm{g}, 0}=2.8 \times 10^{12} \mathrm{~cm}^{-3}\right)$ and three values of laser intensity $\Omega_{0}=(210(\square), 93(\triangle), 42(\circ)) \mathrm{kHz}$. The solid curves are fits to the data with a simple exponential saturation curve. The inset in a shows a magnification of the data in contrast with the calculated Rabi oscillation (dashed) assuming negligible interactions. Fig. c shows a schematic of the excitation dynamics in an inhomogeneous sample. Many oscillating 'superatoms' (shown with exaggerated amplitudes in red) add up to an integrated staturation curve (blue). This curve falls behind the noninteracting case (shown for short times dashed in blue) on a time scale of less than $50 \mathrm{~ns}$.

microsecond time scale. This was done in an echo-type experiment where the excitation dynamics could be reversed [23].

Directly after the excitation pulse the excited Rydberg atoms are field-ionized and the ions detected with a microchannel plate (MCP) detector. The MCP was calibrated and the linearity over the used range verified.

Additionally to the variation of excitation times, we changed the two-photon Rabi frequency $\Omega_{0}$ by changing the power of the $780 \mathrm{~nm}$ excitation laser as well as the initial peak density $n_{\mathrm{g}, 0}$ of the ground state atoms. The latter is done by adiabatically transferring up to $97 \%$ of the atoms with a $6.8 \mathrm{GHz}$ microwave Landau-Zener sweep of variable duration to the untrapped $5 S_{1 / 2}, F=1, m_{F}=1$ state. Due to the large detuning, this state is not affected by the excitation light. With this technique we can vary the peak density with almost no change in temperature and shape of the density distribution. Every excitation and detection of the field-ionized Rydberg atoms is followed by a $20 \mathrm{~ms}$ time-of-flight of the remaining atoms. We take an absorption image of the remaining ground state atoms from which we obtain the ground state atom number. With the temperature and the trapping potential we calculate the density distribution.

Figure2 shows the typical excitation dynamics for three different densities of the ground state atoms (a) and three different Rabi frequencies (b). Two features are prominent in the figure: initially a linear increase with time and a saturation to a constant value. In contrast to previous experiments at considerably lower densities,
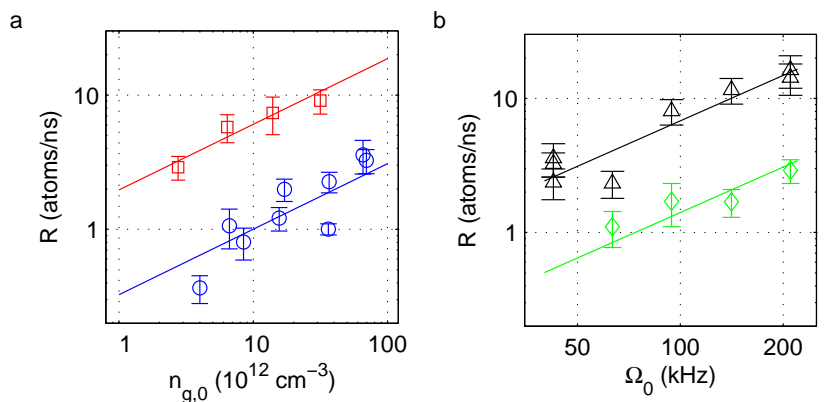

FIG. 3: a Dependence of the initial increase $R$ of the Rydberg atom number on the density of ground state atoms $n_{\mathrm{g}, 0}$ for high $(\square)$ and low (o) Rabi frequency $\Omega_{0}=(210,42) \mathrm{kHz}$. b Dependence of $R$ on the excitation rate $\Omega_{0}$ for high $(\Delta)$ and low $(\diamond)$ atom density $n_{\mathrm{g}, 0}=\left(7.2 \times 10^{13}, 2.8 \times 10^{12}\right) \mathrm{cm}^{-3}$. The lines are the result of a power-law fit to the whole dataset in $\mathbf{a}$ and $\mathbf{b}$ of the form $R \propto n_{\mathrm{g}, 0}^{a} \Omega_{0}^{b}$ which gives an exponent for the $n_{\mathrm{g}, 0}$-dependence of $a=0.49 \pm 0.06$ which is in excellent agreement with the expected $\sqrt{n_{\mathrm{g}, 0}}$-scaling for collective excitation. The fitted exponent for the $\Omega_{0}$-scaling is $b=1.1 \pm 0.1$, which is in good agreement with a linear scaling with $\Omega_{0}$ for coherent excitation.

the dynamics has to be described by full-quantum calculations 24] rather than a mean-field model [2]. However for the following investigations the excitation dynamics curves were fitted with a simple exponential saturation function of the form

$$
N_{\mathrm{R}}(\tau)=N_{\mathrm{sat}}\left(1-e^{-R \tau / N_{\mathrm{sat}}}\right),
$$

with the Rydberg atom number $N_{\mathrm{R}}(\tau)$ after the excitation time $\tau$, since we are mainly interested in the scaling of the saturation Rydberg atom number $N_{\text {sat }}$ and the initial slope $R$. The inset in Fig. 22 contrasts the Rabi oscillation of non-interacting atoms with our measurement and demonstrates the strong blockade of excitation already in the initial linear increase.

Figure 3 a shows the scaling of the initial slope $R$ of the excitation with the density of ground state atoms. In the simplest model, the density $n_{\mathrm{g}, 0}$ is proportional to $N$ (see Eq. 3). For non-interacting Rydberg atoms, the excited fraction would be independent of the atom number and $R$ would scale linearly with $N$. In contrast, $R$ shows a $\sqrt{N}$-scaling, a clear evidence for a collective excitation. Furthermore, we investigated the scaling with the Rabi frequency $\Omega_{0}$ by altering the intensity of the $780 \mathrm{~nm}$ laser. The initial excitation rate of non-interacting atoms or strongly damped (i.e. incoherent) excitation would scale with $\Omega_{0}^{2}$. The linear scaling $\left(R \propto \Omega_{0}\right)$ being determined from Fig. 3b is an evidence for coherent excitation of the Rydberg atoms. The combined $\sqrt{N} \Omega_{0}$-dependence is a clear evidence for local coherent collective Rabi oscillations within a cloud with spatially inhomogeneous density.

The blockade radius depends on the van der Waals in- 


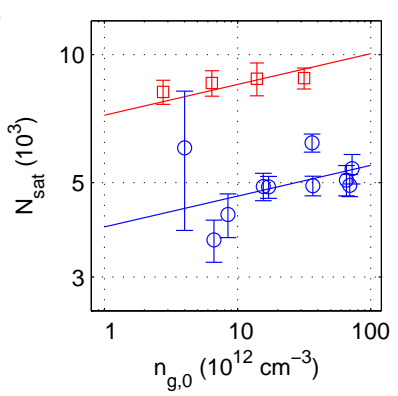

b

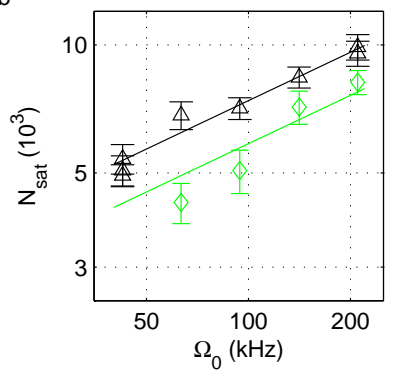

FIG. 4: a Dependence of the saturation number of Rydberg atoms $N_{\text {sat }}$ on the density of ground state atoms $n_{\mathrm{g}, 0}$ for high $(\square)$ and low (o) Rabi frequency $\Omega_{0}=(210,42) \mathrm{kHz}$. b Dependence of $N_{\text {sat }}$ on the Rabi frequency $\Omega_{0}$ for high $(\Delta)$ and low $(\diamond)$ atom density $n_{\mathrm{g}, 0}=\left(7.2 \times 10^{13}, 2.8 \times 10^{12}\right) \mathrm{cm}^{-3}$. The lines are the result of a power-law fit to the whole dataset in a and $\mathbf{b}$ of the form $N_{\mathrm{sat}} \propto n_{\mathrm{g}, 0}^{c} \Omega_{0}^{d}$ which gives an exponent for the $n_{\mathrm{g}, 0}$-dependence of $c=0.07 \pm 0.02$, which is in agreement with the expected independence from $n_{\mathrm{g}, 0}$ for strong blockade. The fitted exponent for the $\Omega_{0}$-scaling is $d=0.38 \pm 0.04$, which is in excellent agreement with the expected $\Omega_{0}^{2 / 5}$-scaling for a collective van der Waals blockade.

teraction strength and the linewidth of the excitation. In our excitation scheme, using cw lasers and large detuning from the intermediate state, the linewidth is dominated by the Rabi frequency $\Omega_{0}$. In the simplest model, we estimate the blockade radius as the distance at which the van der Waals interaction $C_{6} / r^{6}$ equals the power-broadened linewidth $\hbar \Omega_{0}$ except for a geometric factor that includes the arrangement of 'superatoms' (see Fig.1b):

$$
r_{\mathrm{b}} \propto\left[C_{6} /\left(\hbar \Omega_{0}\right)\right]^{1 / 6} .
$$

As the saturation density of Rydberg atoms is proportional to $r_{\mathrm{b}}^{-3}$ and the saturation number of Rydberg atoms $N_{\text {sat }}$ is proportional to their density, $N_{\text {sat }}$ is expected proportional to $\sqrt{\Omega_{0}}$ and independent of the density of ground state atoms. In reasonable agreement with this expectation we observe a very weak $n_{\mathrm{g}, 0}$-dependence in the saturation number of Rydberg atoms as shown in Fig.4 although we change the ground state density by more than an order of magnitude. The average atom number per 'superatom' $N_{\text {mean }}=N_{\mathrm{g}} / N_{\text {sat }}$ is between 65 and 2500, while we expect $N$ to be one order of magnitude higher in the centre of the cloud. Therefore in our experimental setup, using this Rydberg state, a direct observation of single-atom Rabi oscillations is not possible since we would have to reduce the density by a factor of 2500 to about $10^{10} \mathrm{~cm}^{-3}$. This corresponds to a reduction to 4000 atoms which is not possible in a controlled way.

In Fig. 4b the saturation value of the Rydberg atom number is plotted against the single-atom Rabi frequency. The observed dependence is close to the expected $\sqrt{\Omega_{0}}$-scaling which is characteristic for van der Waals in- teraction. Note that in Eq. 3 additional $N$-dependent terms are expected e.g. a $\sqrt{N} \Omega_{0}$-behaviour of the collective Rabi frequency. This closer consideration gives a scaling of $N_{\text {sat }}$ with $n_{\mathrm{g}, 0}^{1 / 5} \Omega_{0}^{2 / 5}$ which is in even better agreement with the experimental observation. Other density dependent effects like number of next neighbours are currently under further theoretical investigation.

To conclude, we have found evidence for mesoscopic quantum dynamics of frozen Rydberg gases in the strong blockade regime. Mesoscopic size effects on the coherent evolution have been identified for up to a few thousand atoms per mesoscopic unit. This became possible by narrowband excitation of magnetically trapped atoms at temperatures of a few microKelvin and variable densities. Analogous size effects are expected in other mesoscopic systems carrying a single excitation quantum only, like an exciton in a quantum dot or a dark state polariton excited by a single photon in an ensemble of atoms [25]. In the latter the time scale for the coherent evolution of the mesoscopic ensemble also speeds up by a $\sqrt{N}$ factor, which is an important factor for quantum repeaters enabling long-distance quantum communication [26]. The demonstrated scalability of the system will enable studies of size dependent quantum correlations and decoherence effects in strongly interacting non-equilibrium situations. In future experiments using Bose-Einstein condensates, phase sensitive measurements beyond mean field might become possible.

We would like to thank Helmar Bender who set up the $6.8 \mathrm{GHz}$ source and Thierry Lahaye for proofreading. We acknowledge financial support from the Deutsche Forschungsgemeinschaft within the SFB/TRR21, SFB407, SPP116 and under the contract PF 381/4-1, U.R. acknowledges support from the Landesgraduiertenförderung Baden-Württemberg.

* Electronic address: r.heidemann@physik.uni-stuttgart.de

$\dagger$ Electronic address t.pfau@physik.uni-stuttgart.de

[1] J. Raimond, G. Vitrant, and S. Haroche, J. Phys. B 14, L655 (1981).

[2] D. Tong, et al., Phys. Rev. Lett. 93(6), 063001 (2004).

[3] K. Singer, et al., Phys. Rev. Lett. 93(16), 163001 (2004).

[4] T. C. Liebisch, A. Reinhard, P. R. Berman, and G. Raithel, Phys. Rev. Lett. 95(25), 253002 (2005).

[5] W. R. Anderson, J. R. Veale, and T. F. Gallagher, Phys. Rev. Lett. 80, 249 (1998).

[6] T. Vogt, et al., Phys. Rev. Lett. 97(8), 083003 (2006).

[7] K. Afrousheh, et al., Phys. Rev. A 73(6), 063403 (2006).

[8] I. Mourachko, et al., Phys. Rev. Lett. 80, 253 (1998).

[9] T. J. Carroll, S. Sunder, and M. W. Noel, PRA 73(3), 032725 (2006).

[10] T. Cubel, et al., PRA 72, 023405 (2005).

[11] J. Deiglmayr, et al., Opt. Commun. 264, 293 (2006).

[12] D. Jaksch, et al., Phys. Rev. Lett. 85, 2208 (2000). 
[13] M. D. Lukin, et al., Phys. Rev. Lett. 87(3), 037901 (2001).

[14] V. Vuletic, Nature Physics 2, 801 (2006).

[15] R. Löw, et al., arXiv:0706.2639 (2007).

[16] W. Li, P. J. Tanner, and T. F. Gallagher, Phys. Rev. Lett. 94(17), 173001 (2005).

[17] A. Grabowski, et al., Fortschr. Phys. 54, 765 (2006).

[18] T. F. Gallagher, Rydberg Atoms (Cambrige University Press, Cambridge, 1994).

[19] B. Knuffman and G. Raithel, PRA 73(2), 020704(R) (2006).

[20] K. Singer, J. Stanojevic, M. Weidemüller, and R. Côté,
J. Phys. B: At., Mol. Opt. Phys. 38, 295 (2005).

[21] W. Li, et al., PRA 70(4), 042713 (2004).

[22] M. P. Robinson, et al., Phys. Rev. Lett. 85, 4466 (2000).

[23] U. Raitzsch, et al., in preparation (2007).

[24] F. Robicheaux and J. V. Hernández, PRA 72(6), 063403 (2005).

[25] M. Fleischhauer, A. Imamoglu, and J. P. Marangos, Rev. Mod. Phys. 77(2), 633 (2005).

[26] L.-M. Duan, M. D. Lukin, J. I. Cirac, and P. Zoller, Nature (London) 414, 413 (2001). 